\title{
Una reseña del desarrollo de la Astronomía en Costa Rica y aportes del Planetario de San José de la Universidad de Costa Rica
}

\author{
Calvo-Gutiérrez, Ivannia \\ Una reseña del desarrollo de la Astronomía en Costa Rica y aportes del Planetario de San José de la \\ Universidad de Costa Rica \\ Revista Educación, vol. 43, núm. 2, 2019 \\ Universidad de Costa Rica, Costa Rica \\ Disponible en: http://www.redalyc.org/articulo.oa?id=44058158003 \\ DOI: https://doi.org/10.15517/revedu.v43i2.29180
}

Esta obra está bajo una Licencia Creative Commons Atribución-NoComercial-SinDerivar 3.0 Internacional. 


\title{
Una reseña del desarrollo de la Astronomía en Costa Rica y aportes del Planetario de San José de la Universidad de Costa Rica
}

\author{
An Overview of the Development of Astronomy in Costa Rica and Contributions by the San Jose Planetarium \\ at the University of Costa Rica
}

Ivannia Calvo-Gutiérrez

Universidad de Costa Rica, Costa Rica, Costa Rica

ivanniacg@gmail.com

http://orcid.org/0000-0002-3367-3258

\author{
DOI: https://doi.org/10.15517/revedu.v43i2.29180 \\ Redalyc: http://www.redalyc.org/articulo.oa?id=44058158003
}

Recepción: 14 Junio 2017

Aprobación: 28 Abril 2019

\section{RESUMEN:}

En este artículo se analiza el desarrollo de la Astronomía, Astrofísica, Ciencias Espaciales y otras áreas de la Ciencia; y el sistema educativo público costarricense y aportes importantes del Planetario de San José de la Universidad de Costa Rica durante una década de su creación; se incluye su relación con la administración de la educación en distintos escenarios históricos, políticos, culturales y sociales costarricenses. También, se hace un breve recuento sobre el nacimiento del curso Fundamentos de Astronomía y su reestructuración; y su correlación con los programas científicos y educativos que se elaboran y exhiben en el Planetario de San José de la Universidad de Costa Rica. Finalmente, se hace un análisis sobre distintas características del Planetario de San José y su conexión con la administración de la educación, según los planteamientos de la Unión Astronómica Internacional (International Astronomical Union [IAU], 2012) y el Programa Estado de la Nación (2014), en el marco de la sociedad costarricense actual.

Palabras clave: Planetario de la Universidad de Costa Rica, Astronomía, gestión de la educación, educación.

\section{Abstract:}

This article describes the development of Astronomy, Astrophysics, Space Science and other areas of Science within the Costa Rican Public Education System and important contributions of the San Jose Planetarium at the University of Costa Rica since its founding ten years ago, and the relationship of the planetarium within the areas of Education Administration as well as the historical, political, cultural and social impact on Costa Ricans. A brief history of the course, Fundamental Astronomy, is analyzed as well as its association with other scientific and educational programs which the Planetarium also offers. Different features of the San Jose Planetarium are analyzed as well as its association with Costa Rica's Public Education Administrative System based on recommendations by the International Astronomical Union [IAU] (2012) and Costa Rica's State of the Nation Program 2014. KEYWORDS: Planetarium of the University of Costa Rica, Astronomy, education management, education.

\section{INTRODUCCIÓN}

Desde la antigüedad, el ser humano se ha planteado por qué ocurren ciertos fenómenos en el firmamento, recurrió a la observación del movimiento del Sol, la Luna y las estrellas, desarrollando la Matemática y la Astronomía; para entender el desplazamiento de los objetos en el cielo. Sin embargo, es en 1609, durante el Renacimiento, cuando el italiano Galileo Galilei utiliza el telescopio por primera vez para observar distintos cuerpos celestes, como los cráteres de la Luna, las lunas de Júpiter, entre otros (Bakulin, Kononovich y Moroz, 1987).

Sin duda, el telescopio constituyó en aquel momento un instrumento clave para entender el movimiento de objetos y observar detalles de la superficie de la Luna y las fases de Venus. De esta manera, otros astrónomos construyeron sus telescopios, pero con ciertas diferencias para observar un objeto en particular, por ejemplo William Herschel descubrió el planeta Urano en 1781 con este instrumento (Bakulin et ál., 1987). 
Según Stephenson, Bolt y Friedman (2000 citados por Taliashvili, 2010) el desarrollo astronómico que se genera durante los siglos XV y XVI impulsa la construcción de sistemas mecánicos que simulan el movimiento de ciertos objetos; de modo que para 1705, George Graham construye un modelo mecánico del Sol, la Luna y la Tierra. Esto evidencia que este sistema mecánico es una aplicación de la ingeniería, para entender el movimiento de estos astros en el firmamento.

En efecto, mientras en algunas partes del mundo se desarrollaban distintas tecnologías que muestran la manifestación de diversas líneas de pensamiento gestadas durante la Ilustración, algunas de ellas tendientes a entender el movimiento de algunos objetos en el cielo; en nuestro país nace, como puntualiza Páez (1994) a través de una comunicación personal de Carlos Meléndez, 100 metros al norte de las Ruinas de Cartago, el fraile Dr. José Antonio de Liendo y Goicoechea.

El fraile José Antonio era hijo de padres españoles, y desde muy pequeño, se traslada a Guatemala, y una vez que los jesuitas salen expulsados en 1767, es De Liendo y Goicoechea quien fomenta la enseñanza de la física experimental y la elaboración de un programa para un curso de física para presentárselo al Rey (Páez, 1994). Se incluían temas como la duda metódica de Descartes, la gravitación universal, experimentos de electricidad o los últimos avances en hidráulica, de tal forma que para 1785, el estudiantado tenía una variada perceptividad de los problemas del conocimiento (Lanning, 1956 citado por Luján, 2008).

Este es un ejemplo de los primeros indicios de la gestión curricular implementada a nivel centroamericano, pues este programa constituyó la base para impulsar la enseñanza de la Física, consolidándola a su vez, como una de las ciencias a través de las cuales se manifiesta la observación meticulosa de fenómenos para explicar la naturaleza que nos rodea.

La predilección del fraile José Antonio por la Matemática y las Ciencias Básicas; se evidencia no solo en el contenido del equipaje que trae de su único viaje a España para fortalecer la biblioteca de la Universidad de San Carlos Borromeo de Guatemala: libros como por ejemplo Diccionario de Física, Elementos de Geometría, Matemáticas y Memoria sobre la Electricidad; este último indudablemente una manifestación del impacto de la Electricidad en la humanidad; sino también al traer equipo como esferas armilares, las cuales se utilizan para explicar el movimiento de la esfera celeste (Páez, 1994). Otro hecho irrefutable de la predilección De Liendo y Goicoechea por la Matemática, la Física y la Astronomía, es palpable en los instrumentos científicos que se encuentran en el escenario en el cual el fraile José Antonio se hace pintar (Páez, 1994).

Tal y como lo expone Jorge Páez, el fraile De Liendo y Goicoechea no solo funge como el primer científico de Centroamérica, sino que también es formador de aquellos involucrados en el proceso de independencia posterior a su muerte en julio de 1814 (Salas, Páez y Herrera; 2013). Evidentemente, en Centroamérica y en nuestro país se manifiesta de alguna manera, la influencia de las ideas que caracterizaron la Revolución Industrial, la Ilustración y la Revolución Francesa, que el fraile José Antonio pudo captar durante su viaje a España, en materia científica, social, política y educativa. En el escenario educativo, la influencia De Liendo y Goicoechea se hace sentir al proponer un programa de un curso de física, como se mencionó anteriormente.

Posterior a De Liendo y Goicoechea, arribarían al país académicos como Juan Rudín y Henry Pittier, entre otros, para fomentar el desarrollo de la Física y la Astronomía en la sociedad costarricense; mediante el uso y perfeccionamiento de laboratorios, telescopios y la implementación de programas de estudio (Salas et ál., 2013), como veremos más adelante en este artículo.

Se evidencia además, un recuento del desarrollo de la Física y la Astronomía en Costa Rica desde la colonia, y que paulatinamente desemboca en la creación del curso Fundamentos de Astronomía, estimulando el interés y el conocimiento astronómico y astrofísico (Páez, 1994); que aumenta aún más con la creación del Centro de Investigaciones Espaciales (CINESPA) y del Planetario de San José de la Universidad de Costa Rica, impulsando el interés y la adquisición de conocimiento astronómico, astrofísico y las ciencias espaciales en general (Taliashvili, 2015).

Luego, como señala Segura (2015), se hace un análisis del impacto del Planetario de San José de la Universidad de Costa Rica en la dinámica educativa, social, cultural, científica y tecnológica costarricense; y 
finalmente, se desarrolla una exhaustiva exploración del rol del Planetario de San José de la Universidad de Costa Rica ante los retos y desafíos del Plan Estratégico 2010-2020 de la Unión Astronómica Internacional [IAU] (International Astronomical Union, 2012) y del Primer Informe sobre el Estado de la Ciencia, la Tecnología y la Innovación 2014 (Programa Estado de la Nación, 2014).

En síntesis, se muestra la Astronomía como una ciencia determinante en distintas épocas costarricenses, y desde la creación del Planetario de San José, se manifiesta con mayor fuerza el interés por la adquisición de conocimiento astronómico, astrofísico y científico de la sociedad costarricense actual.

\section{EDUCACIÓN SUPERIOR Y ASTRONOMÍA EN 1814}

Después del fraile De Liendo y Goicoechea, no hay otra persona que impulsara las ciencias básicas, pero sí algunos personajes ilustres combinados con la manifestación de fenómenos astronómicos (Páez, 1994); como por ejemplo el Bachiller nicaragüense Rafael Francisco Osejo, contratado por la Casa de Enseñanza de Santo Tomás (la cual funcionó de 1814 a 1843) para desarrollar aspectos filosóficos, políticos y científicos (Zelaya, 1971 citado por Páez, 1994); además, Osejo poseía una biblioteca con temáticas sobre Navegación, Astronomía, Óptica, Aritmética e Hidrodinámica, entre otros textos (Páez, 1994).

Otro aspecto importante, es que se manifiestan nuevamente cambios curriculares cuando la Casa de Enseñanza de Santo Tomás para el año 1839, según indican González (1984) y Monge (1978) citados por Páez (1994), incluía los siguientes temas en los exámenes que se aplicaban para optar al título de Bachiller en Filosofía:

1. Lógica: El origen de nuestros movimientos, su atención, hábito y ventajas que resultan de una unión.

2. Física: El lumínico, la propagación y densidad de la luz, la diafanidad, opacidad, colores de los cuerpos, y anillos de Newton

3. Geometría: Diversas especies de líneas, ángulos y método de medidas en general.

4. Astronomía: El equilibrio del Universo, el universo y nombres de los planetas primarios, el tiempo de sus revoluciones, causa de los eclipses y división del globo terrestre: su clima y zonas (p.63)

Para el año 1843, con la fundación de la Universidad de Santo Tomás, se evidencia una reestructuración de la educación superior pública costarricense, pero tal y como lo manifiesta Páez (1994), es en 1849, y según el Ordenamiento establecido por la Reforma de los Estatutos de ese mismo año, se organizó la Facultad de Ciencias, Matemáticas y Física. Esto demuestra la gestión aplicada para fundar la Universidad de Santo Tomás y las primeras señales del nacimiento de la Facultad de Ciencias; además, para 1853 la administración curricular toma mayor fuerza ya que, como indica Páez (1994), el rector de la Universidad, Nazario Toledo, impulsa la reorientación de los estudios en la institución aumentando las materias con un curso de Física Experimental.

En términos de administración de la educación, existen dos puntos de vista importantes de señalar:

a) El primero de ellos, es la contratación de personal de la Universidad. Según Solano y Páez (1991 citados por Páez, 1994), se impulsa la contratación de los profesionales extranjeros como el mexicano Ángel Miguel Velázquez, para atender la carrera de ingeniería, y al francés Luciano Platt, adquiriéndose a la vez los instrumentos para impartir el Laboratorio de Física. Sin duda, se garantiza no solo material humano foráneo que conlleva un enriquecimiento cultural, sino también de equipo de laboratorio que constituye la base del material didáctico y científico que se usó para enseñar Física.

b) El otro aspecto, es el cierre de la Universidad de Santo Tomás en 1888, lo cual constituye un ejemplo de la mala administración de la educación implementada por el Ministro de Educación de entonces, Mauro Fernández, tal y como señala Páez (1994), al manifestar que cerrar la Universidad de Santo 
Tomás, acarreó consecuencias en las generaciones futuras, siendo un error inexcusable en materia educativa.

Con la clausura de la Universidad de Santo Tomás, el equipo de laboratorio adquirido pasa al Liceo de Costa Rica y al Colegio Superior de Señoritas, el cual es utilizado más adelante por Henry Pittier, cuando funge como profesor de Geografía (Salas et ál., 2013).

La adquisición de recursos como equipo de laboratorio y contratación de personal profesional extranjero es una señal de que desde la administración educativa se hace un esfuerzo exhaustivo para reestructurar el sistema educativo público costarricense, ya que se proporciona el material didáctico con el cual el conjunto docente trabajaría de una forma distinta a la tradicional. Además, la influencia extranjera reflejada en el cuerpo docente contratado contribuye a mejorar las lecciones, pues el profesorado llega con ideologías y estructuras de pensamiento diferentes que trasmite a sus estudiantes, de tal manera que aumenta el capital cultural de la época y la criticidad entre el estudiantado que atiende.

No obstante, lamentablemente el cierre de la Universidad de Santo Tomás constituye un ejemplo de la mala función de la administración educativa de la época, pues si bien se atribuye a Mauro Fernández el cierre de la Universidad, también se deben responsabilizar el resto de autoridades relacionadas por permitir tan nefasto error, por cuanto se sesgó el desarrollo del país por un espacio aproximado de 42 años.

\section{Juan Rudín: Pionero en la reestructuración del Sistema educativo público COSTARRICENSE}

Con la finalidad de reestructurar el sistema educativo público en primaria y secundaria, a finales de 1880 el gobierno costarricense gestiona la contratación de varios docentes suizos, entre ellos González (1976) menciona a Juan Rudín, quien nació el 25 de febrero de 1849, en Muttenz, cerca de Basilea, Laussana y Ginebra, Suiza. Rudín tenía una gran formación en Astronomía:

Colaboró con el profesor de matemáticas señor Kinkelin en la elaboración de la estadística escolar de Suiza, obra que se publicó en cinco tomos voluminosos. Tomó parte activa en las conferencias públicas establecidas en la Universidad, cuyas principales disertaciones versaron sobre astronomía. Hizo muchas observaciones con su telescopio particular y realizó importantes publicaciones de sus dibujos y formaciones lunares de planetas y de manchas solares en el periódico astronómico Sirius cuyo redactor, el selenógrafo Kein declaró que sus dibujos eran dignos de ser comparados con los mejores trabajos selenógrafos publicados hasta el día.

Estableció un observatorio popular poniendo a disposición del público un telescopio en días y noches determinadas. La concurrencia a estas observaciones fue numerosa. Obedeciendo a insinuaciones fundó la Sociedad para astronomía particular. Construyó para un buen número de observatorios europeos aparatos astronómicos con sus relojerías correspondientes. (González, 1976, p.281)

Con su arribo, se enriquece el acervo puramente astronómico de nuestro país, y se plasma el impulso que la educación costarricense recibe de un extranjero, pues González (1976) señala que Juan Rudín asume a su llegada al país, el 23 de noviembre de 1889, la dirección del Colegio de San Luis Gonzaga. Desde esta posición, Rudín constituyó un punto de inflexión clave en el desarrollo de la administración de la educación a nivel de primaria y secundaria, tal y como lo expone González (1976) al subrayar que la Inspección General de Enseñanza trabajó para que Juan Rudín se encargara de las clases de pedagogía del Liceo de Costa Rica y el Colegio Superior de Señoritas, y posteriormente, de la dirección técnica de las escuelas de San José, hasta 1903 y se extendió a otras escuelas de la República, tratando de uniformar métodos y procedimientos mediante conferencias celebradas en la Inspección General, con la supervisión de Rudín.

En consecuencia, Rudín no solo impulsa el desarrollo astronómico en nuestro país al prestar a la ciudadanía su telescopio para observaciones, y elaborando material didáctico plasmado en textos como La cosmografía en la Escuela Primaria, El dibujo cartográfico en la Escuela, La Luna y el Cometa Halley; sino que además fomentó los cambios curriculares y metodológicos a nivel de primaria y secundaria (González, 1976). Esto 
evidencia la intervención pertinente y oportuna desde la administración de la educación que implantó Rudín al aplicar su conocimiento para fomentar el desarrollo de la Astronomía y mejorar el sistema educativo público costarricense de la época; lo cual es una manifestación de la gestión, que según Drucker (1999), debe entenderse como: "Proporcionar saber para averiguar en qué forma ese saber existente puede aplicarse a producir resultados es, de hecho, lo que significa gestión" (p.59). Es decir, Rudín aplica distintos conocimientos europeos para generar cambios tanto a nivel astronómico como educativo en la sociedad costarricense atendida durante su gestión.

Así, mientras Juan Rudín y otros ilustres extranjeros contribuyen a mejorar el acervo astronómico y pedagógico costarricense, para el siglo XIX según puntualiza King (1978 citado por Taliashvili, 2010), Wallace Atwood construye el primer prototipo de un planetario tal y como lo conocemos hoy: la denominada Esfera de Atwood para observar el cielo de Chicago en el interior de una esfera de 4,5 metros de diámetro, lo cual impulsa en Alemania, por iniciativa de Max Wolf, que Walter Bauersfeld elaborara el primer simulador del cielo con un proyector óptico electromecánico en el Museo de Múnich; dando paso a la creación del Planetario de Adler de Chicago en 1930.

De esta manera, mientras el primer Planetario de Estados Unidos comienza a funcionar casi al mismo tiempo que la Gran Depresión, en nuestro país nace el pionero de lo que hoy conocemos como el curso repertorio Fundamentos de Astronomía, el Ingeniero Naval Elliot Coen París, a quien nos referimos a continuación.

\section{El Curso Fundamentos de Astronomía}

El 25 de marzo de 1921 nace en Puntarenas, Elliot Coen París, pero a temprana edad se traslada a San José, y una vez finalizados sus estudios de secundaria se muda a Chile para formarse en el área naval (Solano y Páez, 1991). A su regreso en 1948, hizo muchos aportes no solo como Director del Servicio Meteorológico, sino como docente en la Universidad de Costa Rica, primero en la Facultad de Ingeniería y posteriormente, como profesor y jefe de laboratorios del Departamento de Física y Matemática, y además, cabe destacar que, como consecuencia del III Congreso Universitario, desde 1974 el Departamento de Física pasó a ser la Escuela de Física de la Facultad de Ciencias (Fernández, 1994).

El Ingeniero Elliot Coen, en conjunto con Henry McGuie Boyd, Luis González y Renán Méndez, articuló distintas herramientas estratégicas que introdujeron a Costa Rica en el mapa del desarrollo educativo, científico y tecnológico, lo cual dio como resultado que don Elliot Coen impulsara el desarrollo de tres vertientes: la Física como Ciencia, los Laboratorios de Física y la creación de la Radio de la Universidad de Costa Rica (Solano y Páez, 1991).

Como es de suponerse, enumerar y analizar la contribución de Elliot Coen es una tarea sumamente exhaustiva y satisfactoria, considerando que tales aportes se desprenden del espíritu crítico, innovador y emprendedor que siempre lo caracterizó en muchas áreas. Se debe puntualizar que Coen vivió e impulsó muchas de sus ideas, especialmente cuando se lanzó el Sputnik el 4 de octubre de 1957, se descubrió el primer Quasar 3c273 en 1963 y el primer Pulsar en la Nebulosa del Cangrejo, en 1967. Estos eventos, acompañados de la carrera espacial que se vivía durante los sesenta y que desembocó en la llegada del ser humano a la Luna, coinciden con la creación por parte del ingeniero Coen del curso Fundamentos de Astronomía, muy básico, pero formulado desde una perspectiva más formal.

Elliot Coen fue el primer profesor en impartir los cursos H-108 Elementos de Cosmografía en 1964, FM-7107 Fundamentos de Astronomía en 1969 y FS-101 Fundamentos de Astronomía en 1977 (Solano y Páez, 1991). Es claro que Elliot Coen con este curso responde al contexto experimentado por la sociedad costarricense durante las décadas de 1960 y 1970, en materia de Ciencias Espaciales.

Para finales de la década de los ochenta, el curso es impartido por el Dr. Alejandro Sáenz, Dr. Daniel Azofeifa y MSc José Alberto Villalobos, quienes se alternaban para darlo. 
En 1998, quien asume la responsabilidad de impartir el curso es la recién llegada al país procedente de la República de Georgia, la Dra. Lela Taliashvili, y es una prueba más de la influencia profesional extranjera en nuestro país desde distintos puntos de vista, uno de ellos, es el de gestión, iniciándose en materia curricular; ya que el curso adquiere una articulación en las temáticas que aborda todos los tópicos de Astronomía General Básica mediante la utilización de Modelos Clásicos de Astronomía. Para 1999, el curso aumenta a dos grupos, y con esta renovación en el programa del curso, para 2004 se atienden aproximadamente 160 estudiantes matriculados y distribuidos en cuatro grupos. En 2014, la matrícula del curso aumenta a casi 300 estudiantes, distribuidos en cinco grupos (Sistema de Aplicaciones Estudiantiles [SAE], 2015).

En consecuencia, considerando el contexto bajo el cual la Astronomía y otras ciencias se desarrollan en la educación costarricense, partiendo de una iniciativa fomentada por el fraile José Antonio de Liendo y Goicoechea, Juan Rudín y tomando más fuerza a nivel de educación superior, con el aporte docente del Ingeniero Elliot Coen, es evidente que el curso Fundamentos de Astronomía, catalogado como repertorio, para finales de los años 1990, constituye una articulación pertinente de los contenidos que se deben abarcar, respondiendo a demandas académicas y contextuales, tal y como se sintetiza en la siguiente Figura 1.

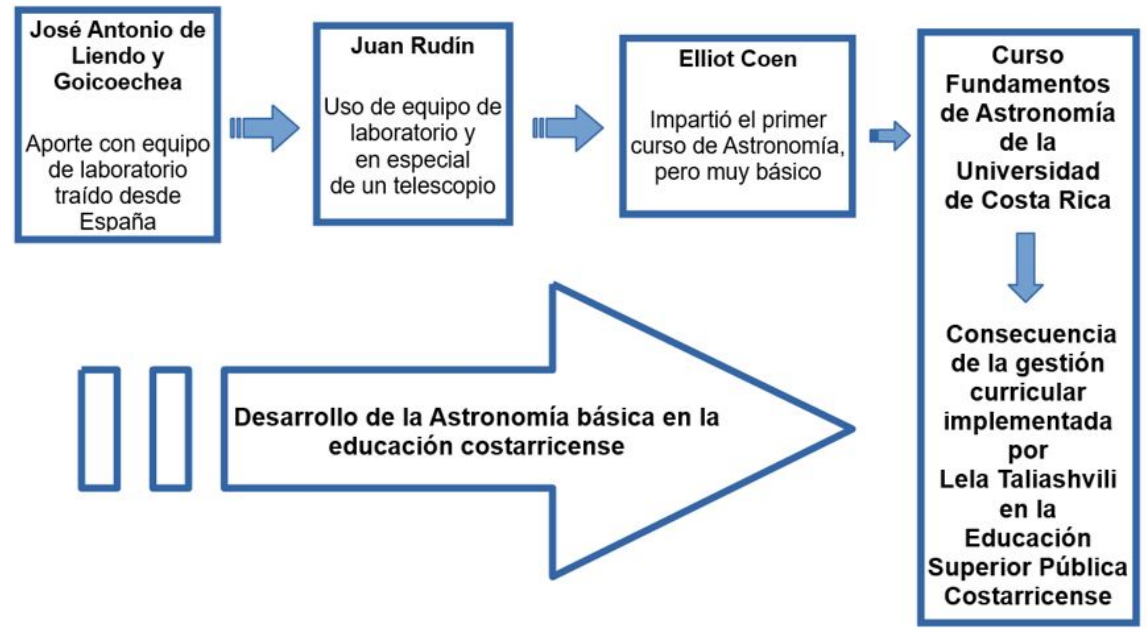

FIGURA 1

La Astronomía en la educación costarricense

Fuente: Elaboración propia, 2016

\section{El Planetario de San José de la Universidad de Costa Rica}

Una vez que el curso Fundamentos de Astronomía adquirió una renovación en el desarrollo de sus contenidos, para setiembre de 1997 nace el Laboratorio de Investigaciones Astrofísicas (LIA), un año después la Sección de Astronomía y Astrofísica; y para el año 2002 culmina con la creación del Centro de Investigaciones Espaciales CINESPA, finiquitándose al mismo tiempo la donación por parte del gobierno japonés de un sistema de proyección mecánico óptico: un domo de 8,5 metros de diámetro, y un proyector GOTO GS. La construcción del edificio inició en 2004 y el 8 de julio de 2005 se inaugura el Planetario de San José de la Universidad de Costa Rica. Para este momento, se contó con la colaboración del director del Planetario de Medellín, Colombia, el Dr. Gabriel Gómez, quien contribuyó en la elaboración de los primeros programas astronómicos del Planetario de San José.

A 10 años de su creación, inicialmente como un Proyecto y desde 2012 como el Programa de Educación y Divulgación Científica (Programa-Planetario), el Planetario de San José ha jugado una función trascendental en el contexto en el cual se está desarrollando la Astronomía y Astrofísica en nuestro país, colaborado y participando en eventos y/o particularidades como por ejemplo la celebración del Año Internacional de la 
Astronomía en el año 2009, conmemorando 400 años desde que Galileo apuntó por vez primera al cielo con un telescopio (Taliashvili, 2009).

Debido a lo anterior, el Planetario de San José constituye un referente único profesional de consultas astronómicas, y una fuente desde donde se expanden distintos tópicos y temáticas en torno a la Astronomía como se visualiza actualmente; lo cual conduce sin duda alguna, a la reflexión de distintas áreas científicas, tecnológicas, educativas, sociales, culturales, económicas y políticas que convergen en el Planetario de San José (Segura, 2015).

En consecuencia, tal y como lo manifiesta Fernández (2008), la reestructuración que implementó la Dra. Taliashvili desde el año 2006 como coordinadora del curso Fundamentos de Astronomía, así como la forma bajo la cual ha administrado y dirigido el Planetario de San José de la Universidad de Costa Rica, la consolida sin duda, como un ejemplo más de la influencia profesional en el desarrollo científico, tecnológico y educativo costarricense. Por lo tanto, en virtud del contexto en el que se ha desarrollado el Planetario de San José desde su nacimiento a la fecha, es importante considerar la función de la gestión, que según Drucker (1999) se aplica no solo a empresas, sino también a instituciones y/o organizaciones que no son un negocio, más bien cumplen una función social.

Evidentemente, el Planetario de San José es una organización que aplica prácticas de gestión tal y como lo puntualiza Drucker (1999), al manifestar que provee conocimiento para determinar en qué forma el conocimiento existente se aplica para generar resultados. Constantemente, se debe indagar como organización todos aquellos nuevos saberes que fortalezcan los ya existentes en materia astronómica, administrativa, científica, tecnológica y educativa; y que desemboquen en brindar un mejor servicio a quienes visitan el Planetario de San José.

Sin duda, aplicar el concepto de gestión de Drucker (1999), implica estar abierto al cambio en todas las áreas, y en este proceso, es evidente que una persona que ha dirigido una organización como el Planetario de San José durante más de 10 años, contribuye a generar conocimiento tanto en el marco de la administración educativa como en el astronómico y educativo, aumentando el capital cultural de la organización y de quienes lo visitan de una forma innovadora, eficiente, dinámica y versátil; mediante la implementación de una política de puertas abiertas, de modo que el conocimiento generado en el Planetario de San José en todas las áreas, sea accesible a la mayor cantidad de la población costarricense.

\section{Impacto del Planetario de San José de la Universidad de Costa Rica en la dinámica EDUCATIVA, SOCIAL, CULTURAL, CIENTÍFICA Y TECNOLÓGICA COSTARRICENSE}

El nacimiento del Planetario de San José constituye un factor sumamente importante para fomentar desde un marco profesional y especializado, el interés por la Astronomía y las Ciencias Espaciales en general, a través de sus programas y actividades educativas de divulgación científica, los cuales abarcan algunos contenidos ya sistemáticamente establecidos en el curso Fundamentos de Astronomía:

A diferencia, los planetarios universitarios o profesionales se encuentran en observatorios y universidades y con su equipo, principalmente de científicos y profesionales y se enfatizan en múltiples actividades didácticas y de divulgación científica, como son programas educativos, material didáctico impreso y audiovisual, cursos y talleres de capacitación, conferencias científicas, seminarios y observaciones nocturnas, hacen de ellos, centros científicos destacados en la divulgación científica y enseñanza en Astronomía, aunque también ofrecen una variedad de la programación cultural. (Taliashvili, 2015, p.13)

Aunado a lo anterior, el Planetario de San José también nace cuando las Tecnologías de la Información y la Comunicación (TIC) están en su máximo apogeo, lo cual facilita el contacto entre los funcionarios del Planetario visto como una organización, y con quienes lo visitan; lo cual conduce al establecimiento de una comunicación interactiva cuando ocurre un fenómeno astronómico que captura el interés y la curiosidad de la población en general, como eclipses, ocultaciones, lluvia de meteoros, entre otros. 
Esto adquiere mayor fuerza, desde el año 2014, con la adquisición del proyector digital Fulldome, dispositivo empleado para proyectar documentales y películas de divulgación científica abarcando $360^{\circ}$ de amplitud.

Además, existe en la actualidad una gran cantidad de sitios en Internet que se caracterizan por tener información astronómica para todas las edades; pero el acceso no garantiza un aprendizaje, pues se requiere cierto conocimiento y orientación educativa para entender y comprender correctamente, de modo que los funcionarios del Planetario de San José son un factor clave para contribuir en la orientación de la adecuada comprensión de este conocimiento. Por eso, los funcionarios que se encargan de proyectar la programación son jóvenes estudiantes que cursan el bachillerato en Física y/o la Maestría en Astrofísica, además, dos de ellos tienen una formación en Enseñanza de la Física y/o Ciencias de la Educación (Taliashvili, 2015). Aunado a esta formación, los funcionarios tienen las bases temáticas profesionales con respecto a Astronomía y Astrofísica, en función de la población que atienden.

En virtud de lo anterior, es sumamente importante recalcar que la metodología empleada por el Planetario de San José, consiste en proporcionar a los colaboradores del Planetario -principalmente han sido estudiantes de la Escuela de Física y de la Facultad de Educación interesados en Astronomía- un exhaustivo y excelente asesoramiento en el uso de los proyectores GOTO y Fulldome, ambos exclusivos de un planetario profesional, así como también en Astronomía Observacional y educación astronómica (Taliashvili, 2015).

Lo anterior, fortalece la formación y el conocimiento científico sobre Astronomía, Astrofísica y Educación del personal de Planetario de San José de la Universidad de Costa Rica, para generar un ambiente de divulgación científico-educativa, plasmada en la preparación de material didáctico original y actualizado, basado en artículos científicos, observatorios espaciales y terrestres para producir películas educativas, videos, afiches, calendarios astronómicos, entre otros. Esto requiere la implementación de distintas herramientas metodológicas que trasciende la labor que se desarrolla en una clásica lección de Ciencias, Física y afines de diferentes niveles educativos.

Por eso, es importante considerar el estudio elaborado por Plummer y Small (2013), del cual se desprenden seis perspectivas o puntos de vista, que en adelante llamaremos lens, cada uno desde los cuales el funcionario de un planetario genera un ambiente de aprendizaje idóneo, que puede estar centrado en: el aprendizaje (lo cual comprende la construcción de conocimiento previo, interacción con las experiencias de otros visitantes al planetario, y proporcionando herramientas para un futuro aprendizaje), el conocimiento (focalizado en mejorar el entendimiento del contenido científico que se trata de transmitir), en la evaluación (muchos funcionarios discuten el diseño de un ambiente centrado en la evaluación considerando los tipos de visitantes que llegan al planetario), en la motivación (focalizado en tres perspectivas: aumentando el interés del público por la Astronomía, fomentar el aprendizaje y aumentar el interés más allá de lo que se percibe en el planetario), la parte sociocultural (se consideran teorías socioculturales las cuales contribuyan a entender cómo las interacciones sociales influyen en aprendizaje en museos y afines) y en lo físico (enfatiza al Planetario como un lugar en el que se proyecta un programa o una película especial asociada a una proyección de un cielo artificial simulado).

Estos seis factores propuestos y estudiados por Plummer y Small (2013), se manifiestan y contextualizan en el Planetario de San José de la Universidad de Costa Rica de la siguiente manera:

1. Centrado en el aprendizaje: Este punto de vista se refiere a focalizar la atención y el aprendizaje en estudiantes de diferente nivel educativo y/o de cualquier persona que visite el Planetario de San José mediante el constructivismo, el cual se explota en su máxima expresión durante uno de los programas denominado Joyas de Firmamento, pues hay interacción entre el funcionario profesional responsable de la función y las personas que asisten. Por lo tanto, esta interacción es fundamental en un enfoque centrado en el estudiantado (Plummer y Small, 2013).

Este enfoque adquiere mayor fuerza durante el desarrollo de distintas actividades adicionales del Planetario, como telescopiadas, conferencias, cursos y talleres; durante las cuales es inminente que 
se establece una interacción entre el funcionario profesional a cargo y quien esté participando de alguna de las actividades anteriormente mencionadas.

2. Centrado en el conocimiento: Este es el enfoque o el punto de vista que se manifiesta con mayor y particular interés en el Planetario de San José, ya que todos los programas tienen carácter de divulgación científica actualizada y de acuerdo a la edad de quienes asisten, se canaliza a través de recursos multimediales; elaborados por el personal del Planetario de San José debidamente capacitado. No se trata de dictar conceptos, sino una síntesis de conocimientos que contribuya a que la persona que observa el programa educativo, adquiera un entendimiento integral, sin que eso signifique superficialidad. Así, centrarse en el conocimiento es mucho más importante que el contenido deseado por los visitantes (Plummer y Small, 2013).

Con los distintos programas educativos se exponen y abarcan múltiples áreas de Astronomía, Astrofísica y Ciencias Espaciales, y combinados con las actividades relacionadas a la dinámica que caracteriza al Planetario de San José; el personal profesional con su conocimiento impulsa el interés, la criticidad y la comprensión del firmamento; lo que a su vez desemboca en la divulgación científica y produce que el Planetario de San José, como organización, sea un medio a través del cual el visitante entienda y aprenda sobre distintos fenómenos y procesos físicos, y planteamientos matemáticos correctamente. Es decir, el Planetario de San José constituye un ente a través del cual un visitante entiende, capta y se acerca a la realidad de un fenómeno y/o proceso astronómico naturalmente, lo cual es difícil lograr en un salón de clase.

En el caso del Planetario de San José, la idea es colaborar para que los visitantes adquieran y/o fortalezcan desde un punto de vista informal, los conocimientos científicos plasmados en los contenidos y objetivos de los Programas de Ciencias del Ministerio de Educación Pública estructurados para todos los niveles (Ministerio de Educación Pública [MEP], 2003, 2012, 2014a, 2014b).

3. Centrado en la Evaluación: Plummer y Small (2013) no se anticipan a estudiar el diseño de un entorno de aprendizaje centrado en la evaluación, debido a la naturaleza transitoria de las audiencias que visitan planetarios; sin embargo, proponen tres opciones:

- Formas generales de evaluación, en donde la evaluación es parte de la práctica o labor del funcionario: En este punto de vista, el Planetario de San José coincide con Plummer y Small (2013) cuando en uno de los programas, hay una persona exponiendo directamente, y quien puede plantear preguntas y respuestas a la audiencia tendientes a debates. La evaluación, aunque es de carácter formativo y se realiza pocas veces (debido a la escasez de tiempo del estudiantado y personal docente de distintos centros educativos), ha mostrado excelentes resultados.

- Formalizar formas de utilización de la evaluación: Desde esta perspectiva, el director de un Planetario propone que se diseñen preguntas sistemáticamente establecidas, pero no con la finalidad de nivelar a las personas que asisten al planetario, sino para regresar de nuevo a un determinado concepto, y verificar si se entendió la temática o tópico estudiado (Plummer y Small, 2013). Esta opción se sustenta normalmente al final de todas las programaciones educativas del Planetario de San José, pues algunos miembros de la audiencia plantean preguntas cuyas respuestas se fortalecen aún más con los recursos mutlimediales que están fuera de la sala

- Nueva evaluación sobre el aprendizaje en el planetario; ya que algunos profesionales de planetarios, reconocen que la evaluación es una forma de valorar si están cumpliendo o no con los objetivos, pero algunos creen que aún queda trabajo por hacer (Plummer y Small, 2013). Esta premisa debe fortalecerse en el Planetario de San José, específicamente con las películas educativas, de modo que los distintos conceptos que se visualicen no den lugar a 
dudas o vacíos cognitivos. También, es importante impulsar las visitas guiadas después de las proyecciones, con la finalidad de complementar lo que se observa en la sala. Esperemos que en un futuro cercano el Planetario de San José tenga más colaboradores para lograr esta meta.

4. Orientado a la motivación: Este aspecto coincide perfectamente con el Planetario de San José, pues Plummer y Small (2013) manifiestan una orientación hacia engendrar y aumentar el interés entre sus audiencias; y se expresa en tres direcciones:

- un deseo general de aumentar el entusiasmo o interés de su público

- el deseo de aumentar el interés, y el compromiso de fomentar el aprendizaje

- según el tema, el deseo de aumentar el interés por lo que el aprendizaje se extenderá más allá de la experiencia del planetario

En el Planetario de San José se abarcan las tres categorías, lo cual se evidencia en el aumento en el número de estudiantes que matriculan el curso Fundamentos de Astronomía, el cual desde el 2014 aumentó a 5 grupos (SAE, 2015). Además, los profesionales que laboran en el Planetario, desarrollan un particular interés no solo en temáticas afines a la Astronomía y Astrofísica, sino también en otras áreas como la programación computacional científica, organización de conciertos Música bajo las estrellas en colaboración con el Trabajo Comunal Universitario TCU, manipulación de imágenes astronómicas usando software libre como: celestia, stellarium, blender y gimp, entre otros; lo que a su vez consolida al Planetario de San José como una organización impulsora, por su dinámica de trabajo, el desarrollo de la multidisciplinariedad, porque permite el mejoramiento de distintas habilidades y destrezas de los profesionales que lo integran.

5. Orientado a lo sociocultural: Existen teorías socioculturales en el aprendizaje que nos pueden ayudar a comprender cómo las interacciones y el contexto influyen en el proceso de aprendizaje de museos y centros de ciencia, de modo que un planetario deje de percibirse como lo dicen Davidson, Passmore, y Anderson (2010); Tal y Morag, (2007) (citado por Plummer y Small, 2013); un escenario pasivo donde se exhiben películas educativas, sino que además, sea un lugar en el que se facilite el aprendizaje adquirido durante un programa, por medio de interacciones con el profesional encargado cuando es requerido (Plummer y Small, 2013).

En el Planetario de San José, el rol del funcionario profesional es desde un inicio, dar las pautas que se usarán durante la proyección educativa, pero a la vez se proporciona una breve descripción de la temática que se observará; y es durante esta interacción, donde el profesional del Planetario de San José determina la calidad del conocimiento astronómico y el interés que tienen las personas que visitan el Planetario, y en función de este conocimiento o interés, se hace un cierre al finalizar la programación educativa (Taliashvili, 2015). Esto es sumamente importante, por cuanto es la base sobre la cual se planifica la elaboración de documentales y películas de divulgación científica a corto y mediano plazo (Segura, 2015).

En consecuencia, la interacción social, para quienes proyectan la función, es una forma de aumentar el interés en un tema, y mejorar la calidad del conocimiento del contenido (Plummer y Small, 2013). De modo que al entablar contacto, tal y como lo señalan Davidson, Passmore y Anderson (2010) y Griffin y Symington (1997) (citado por Plummer y Small, 2013), la audiencia suele plantear preguntas cuyas respuestas esperan del profesional ISE [Informal Science Education o Educación Científica Informal en español], de modo que es una dinámica que refuerza las recomendaciones de investigadores sobre el aprendizaje en ambientes informales (Plummer y Small, 2013). En este punto llama la atención cómo se produce una realimentación entre los visitantes y el profesional responsable de la proyección; lo cual desemboca en impulsar y fomentar el interés por la Astronomía, la Astrofísica y las Ciencias Espaciales en general, y evidentemente 
le da a esta forma de aprendizaje, otra connotación: no se trata solo de proyectar el programa educativo, sino también de generar escenarios de aprendizaje que tiendan a la criticidad, a partir de los conocimientos que nacen de la proyección del programa educativo y del tipo de visitantes que arriban al Planetario de San José que suelen conformar, según señala Taliashvili (2012), los siguientes grupos:

- estudiantes y docentes de todos los niveles y de diversas clases sociales, tratando de abarcar los centros educativos de Atención Prioritaria.

- docentes y autoridades universitarias, que pueden ser de la Universidad de Costa Rica o de otras instancias.

- público general

En este sentido, es evidente que la calidad del aprendizaje que adquiere cada uno de estos individuos y grupos, representa un reto para el profesional que atiende la proyección del programa educativo, pues son distintas personas y cada una de ellas tiene un acervo cultural y social distinto y definido; que en ocasiones conduce a replantear los más elementales cuestionamientos en torno al origen de objetos como la Luna y la Tierra; y es en este punto donde la interacción social y cultural adquiere su máxima expresión.

6. Orientado a lo físico: Desde este ángulo, un planetario es un teatro en el que la experiencia está relacionada con la proyección simulada del cielo (Plummer y Small, 2013). De esta forma, es evidente que el interés por las Ciencias Espaciales, la Astronomía y la Astrofísica, se propaga desde el Planetario de San José con más fuerza que nunca, lo cual se incrementa con distintas herramientas informativas adicionales: la observación en 3D de Marte, de la Estación Espacial Internacional [International Space Station, ISS] en una pantalla plana, y de manchas solares por medio de telescopios, si está despejado.

Desde el año 2015, esta motivación se ha consolidado con la implementación del proyector digital fulldome de $360^{\circ}$ de amplitud, con el cual se obtiene una proyección en toda la cúpula del domo durante las presentaciones y animaciones astronómicas (Taliashvili, 2015). Esta nueva tecnología innovadora permite proyecciones de los objetos y los fenómenos del universo con sensaciones de profundidad y de realidad virtual.

Tal y como lo exponen Plummer y Small (2013) en los seis lens anteriores, en el Planetario de San José de la Universidad de Costa Rica se han generado distintos espacios que han propiciado diferentes puntos de debate, discusión, sugerencias y/o cuestionamientos en áreas diversas que han impulsado el análisis relacionado con: a) inquietudes sobre las características del telescopio que una persona puede adquirir, b) surgen distintas ideas para ferias científicas educativas institucionales y c) dudas sobre el objeto celeste que se está observando.

Este tipo de espacios conduce al profesional responsable de la programación, a interactuar con el visitante, antes o después de la programación; sin embargo, el programa educativo original en donde hay una interacción flexible es Joyas del firmamento, ya que es una narración en vivo que proyecta la esfera celeste para el día específico de la proyección, y se recrea la observación de algunos objetos usando binoculares o telescopios. Además, las observaciones nocturnas o telescopiadas generan excelentes condiciones de interacción entre visitantes y astrónomos profesionales.

Debido a esta interacción con los visitantes, se ha detectado que es importante mejorar los conocimientos astronómicos de la población costarricense, cuyos contenidos se reflejan en los programas de estudio de Ciencias de la Educación General Básica y de Física de la Educación Diversificada del Ministerio de Educación Pública, pero por la estructura fraccionada y segmentada que tienen, no impulsan ni propician la relación de un concepto con otro. 
Por ejemplo, según el Programa de Estudio de Ciencias, durante el primer ciclo de la educación general básica, se estudian los movimientos que realizan la Tierra y la Luna, la rotación de la Tierra sobre su propio eje, la traslación de la Luna alrededor de la Tierra, el peso y la atracción gravitatoria, así como algunos componentes del sistema solar: estrellas, planetas, satélites, asteroides, cometas y meteoros (MEP, 2014a); mientras que en el segundo ciclo de educación general básica se estudian las fases de la luna, los eclipses lunares y solares, los inicios de la exploración espacial y algunos componentes del Universo: estrellas y cúmulos estelares, galaxias y sus tipos, nebulosas, constelaciones más brillantes (MEP, 2014b).

A nivel de secundaria, durante sétimo año se estudia la aceleración de la gravedad, movimientos de la Tierra, los planetas del sistema solar, las leyes de Kepler y colisiones de asteroides y cometas en el sistema solar (MEP, 2012); mientras en la educación diversificada se vuelve a hacer referencia a los contenidos relacionados con las leyes de Kepler, movimiento de planetas y de satélites en órbitas circulares, para finalizar con la ley de Gravitación Universal (MEP, 2003).

Es decir, si bien desde un inicio los contenidos estudiados son muy básicos, se tornan repetitivos, y no se establece una relación o articulación de un concepto con otro; por lo tanto, en el Planetario de San José de la Universidad de Costa Rica los docentes tienen una oportunidad de ampliar y sintetizar sistemáticamente de una forma innovadora y dinámica lo que se estudia en una clase típica de Ciencias.

Además, las películas educativas actualizadas de divulgación científica que el personal profesional del Planetario de San José elabora, constituye un apoyo fundamental para que el cuerpo docente fortalezca y actualice sus conocimientos en materia astronómica, tal y como lo refleja la tesis para optar al grado de licenciatura de Ana Yancy Segura Zárate titulada: Propuesta didáctica para fortalecer la enseñanza de la Astronomia, a nivel deprimaria en Costa Rica, según programa de Ciencias del Ministerio de Educación Pública, vigente en el año 2015. En esta investigación, se evidencia que el Planetario de San José es una base para desarrollar distintos tipos de estudios académicos, y también prueba que el material audiovisual educativo elaborado constituye un apoyo fundamental para entender distintos tópicos astronómicos que el profesorado (por falta de tiempo y/o conocimiento), no desarrolla en clase:

En segundo lugar, se logra diseñar y crear una propuesta didáctica en el tema del Sistema Solar. Esta propuesta conlleva trabajo de aula previo y posterior a una película educativa que permite ahondar y tener conocimiento científico fidedigno y actualizado, debido a que según la investigación hecha, es una de los mejores métodos de enseñanza ya que la población infantil absorbe mejor los conocimientos cuando escuchan y ven algún tema en específico, y este se puede consolidar aún mejor si se discute en clases y si además, realizan alguna actividad relacionada con dicho tema. Para llevarla a cabo, se trabajó en conjunto con personal del Planetario de la Universidad de Costa Rica.

Este material audiovisual explica las principales características de nuestro Sistema Solar, cada uno de los cuerpos que lo componen, sus movimientos y sus particularidades, obtenidas de las distintas misiones espaciales. (Segura, 2015, p.78)

Ante este escenario, el Planetario de San José es un recurso sumamente pertinente y trascendental, ya que contribuye ampliando los contenidos que serán evaluados, aumentando el capital cultural de quienes lo visitan, a través del material didáctico que ha generado (como calendarios astronómicos y películas astronómicas de divulgación científica). Al mismo tiempo, el Planetario de San José ha sido una base para desarrollar trabajos finales de graduación, proyectos de docencia, de investigación y de acción social.

En consecuencia, el material didáctico audiovisual producido a través de la implementación de software libre y de la información actualizada de las más recientes misiones espaciales, se ha obsequiado a distintas bibliotecas de centros educativos, y los programas educativos se han donado también a otros planetarios latinoamericanos interesados.

Paralelamente, es importante recalcar que los colaboradores del Planetario, que principalmente han sido las y los estudiantes de la Escuela de Física y de la Facultad de Educación interesados en Astronomía, han recibido un excelente asesoramiento en Astronomía Observacional y educación astronómica, lo cual los ha motivado a seguir sus estudios de posgrado en Astronomía y Astrofísica.

Además de las proyecciones y las visitas, se han desarrollado otras actividades como: 
a) Noches de observación Telescopiadas: Durante estas noches de observación, se atienden consultas de los objetos observados, y además, de las características que debe tener un telescopio.

b) Talleres astronómicos: Estos se combinan con el Trabajo Comunal TC-535: Astronomía y sus aplicaciones, durante los cuales se desarrollan talleres que abarcan distintas temáticas como: sistema solar, nebulosas, cohetes, galaxias y relojes solares, entre otros.

c) Conciertos de Música bajo las estrellas: También combinado con el Trabajo Comunal antes citado, se proyectan imágenes astronómicas acompañadas con música en vivo, con la participación especial de estudiantes de la Escuela de Artes Musicales de la UCR.

d) Cursos astronómicos: Estos cursos son impartidos por profesionales, y abarcan astronomía observacional, conceptos básicos, visión del Universo y discusiones con expertos en Astronomía.

e) Conferencias: Las conferencias son impartidas por profesionales en Astronomía y Astrofísica, de prestigio nacional e internacional, sobre distintos temas como: Física solar, Lentes gravitacionales, Astronomía extragaláctica, Radioexplosiones solares, Relatividad Espacial, Rayos Cósmicos, entre otros.

En consecuencia, las actividades anteriores han propiciado una interacción dinámica con los visitantes, y, en consecuencia, han promovido el interés por la Astronomía y la adquisición de conocimiento.

Aunado a lo anterior, el Planetario de San José ha coordinado con distintas organizaciones para programaciones específicas, asesoramiento a la prensa y público en general en distintas temáticas y/o eventos astronómicos, como cuando ocurren fenómenos como eclipses, ocultaciones, paso de asteroides y otros. Es evidente que el Planetario de San José es una organización que constituye una herramienta estratégica de la Universidad de Costa Rica que responde de una forma innovadora al contexto científico-tecnológico desafiante actual, que se analizará en el siguiente apartado.

Rol del Planetario de San José de la Universidad de Costa Rica ante los retos y desafíos del Plan Estratégico 2010-2020 de la Unión Astronómica Internacional y del Primer Informe sobre el Estado de la Ciencia, la Tecnología y la INNOVACIÓN 2014

El escenario político, económico, educativo, cultural, social, científico y tecnológico actual constituye un reto para la sociedad costarricense, en aras de impulsar el proceso de desarrollo de nuestro país en todas las áreas posibles; por lo cual es impostergable articular la dinámica política y el trabajo científico, tal y como lo señala el Primer Informe sobre el Estado de la Ciencia, la Tecnología y la Innovación (2014) al puntualizar: "El país requiere potenciar las coincidencias entre las prioridades de la política pública y las áreas robustas del quehacer científico, para lograr encadenamientos claros y sostenibles de esos grupos más dinámicos con los sectores productivo y social" (Programa Estado de la Nación, 2014, p.20).

Bajo este contexto, el Planetario de San José como organización, constituye una herramienta estratégica trascendental, por cuanto provee de las herramientas innovadoras requeridas por medio de las cuales el ejercicio docente impulsa su trabajo de aula y la divulgación científica, fortaleciendo la temática estudiada en clase y distintas áreas del conocimiento, respondiendo así a distintos objetivos y parámetros plasmados en el Plan Estratégico 2010-2020 propuesto por la Unión Astronómica Internacional (IAU, 2012), al subrayar la Astronomía como una combinación única de ciencia, tecnología y cultura; lo que la convierte irrevocablemente en un punto de inflexión importante en la sociedad actual por varias razones, según el mencionado Plan Estratégico como las siguientes:

- El universo proporciona un laboratorio económico para el estudio de condiciones extremas que son inaccesibles en la Tierra. Las estrellas y las galaxias son ambientes que han producido elementos químicos y moléculas orgánicas, componentes básicos de la vida. Durante estudios astronómicos del 
siglo pasado han tenido lugar nuevos descubrimientos en física, química y biología; desembocando en nuevas ciencias como astrofísica, astrobiología y astroquímica. Además, debido a su base matemática, la astronomía es también una excelente herramienta para la enseñanza de la matemática.

- La astronomía ha sido un factor importante para el desarrollo de tecnología avanzada, como los detectores más sensibles de luz y ondas de radio, así como las computadoras más rápidas. La necesidad de estudiar los objetos más débiles requiere una electrónica sofisticada y de extrema precisión óptica adaptativa, así como ingeniería del estado de la técnica. La astronomía también ha desempeñado un papel importante en el desarrollo de la tecnología espacial que se ha abierto al Universo, para el estudio en todo el espectro electromagnético. Radiotelescopios y óptica moderna son algunos de los equipos más avanzados jamás construidos, y son un mecanismo educativo para la introducción de la última tecnología compleja. (IAU, 2012, p.15)

Aunado a lo anterior, es indispensable subrayar que, al ser la Astronomía y la Astrofísica fuente del saber humano, evidentemente constituye un área del conocimiento a través del cual, un individuo adquiere la capacidad de desarrollar la observación y la experimentación, y por lo tanto, del método científico, lo cual a su vez produce como resultado, un exhaustivo y constante aumento de interés y conocimiento por la Astronomía y Ciencias en general; y paralelamente, contribuye al desarrollo cultural desde varias perspectivas, según se puntualiza en el mencionado Plan Estratégico 2010-2020:

- Debido a que la radiación del universo distante tarda tanto en llegar a la Tierra, un astrónomo puede sondear profundamente en nuestro pasado. Grandes telescopios que operan en todo el espectro electromagnético son máquinas del tiempo que habitualmente proporcionan imágenes y otros datos acerca del Universo observable cerca de su nacimiento, hace 13,7 mil millones de años. Desenredar la historia del Universo ha sido un logro de la humanidad durante el último medio siglo.

- Una de las funciones sociales más importantes de la astronomía moderna es que constituye una herramienta para la educación en el sentido más amplio. Debido a que es una de las ciencias más accesibles, y que fascina constantemente a los jóvenes, la astronomía es un excelente vehículo para introducir a los niños y niñas a la ciencia y la tecnología. La accesibilidad del cielo, la belleza cósmica de los objetos y la inmensidad del Universo son fuente de inspiración y proporcionan una perspectiva que alienta el internacionalismo y la tolerancia. La pasión de la astronomía ha estimulado un gran número de jóvenes que eligen una carrera relacionada a la ciencia y la tecnología, lo que contribuye a la economía del conocimiento de muchos países. (IAU, 2012, p.16)

Sin duda, la pasión por la astronomía implica fortalecer áreas culturales, científicas y tecnológicas, que desemboquen en una inminente articulación y desarrollo en distintos campos: educativos, culturales, científicos, económicos, políticos y tecnológicos; con el evidente beneficio a la sociedad costarricense actual, como se visualiza en la siguiente Figura 2: 

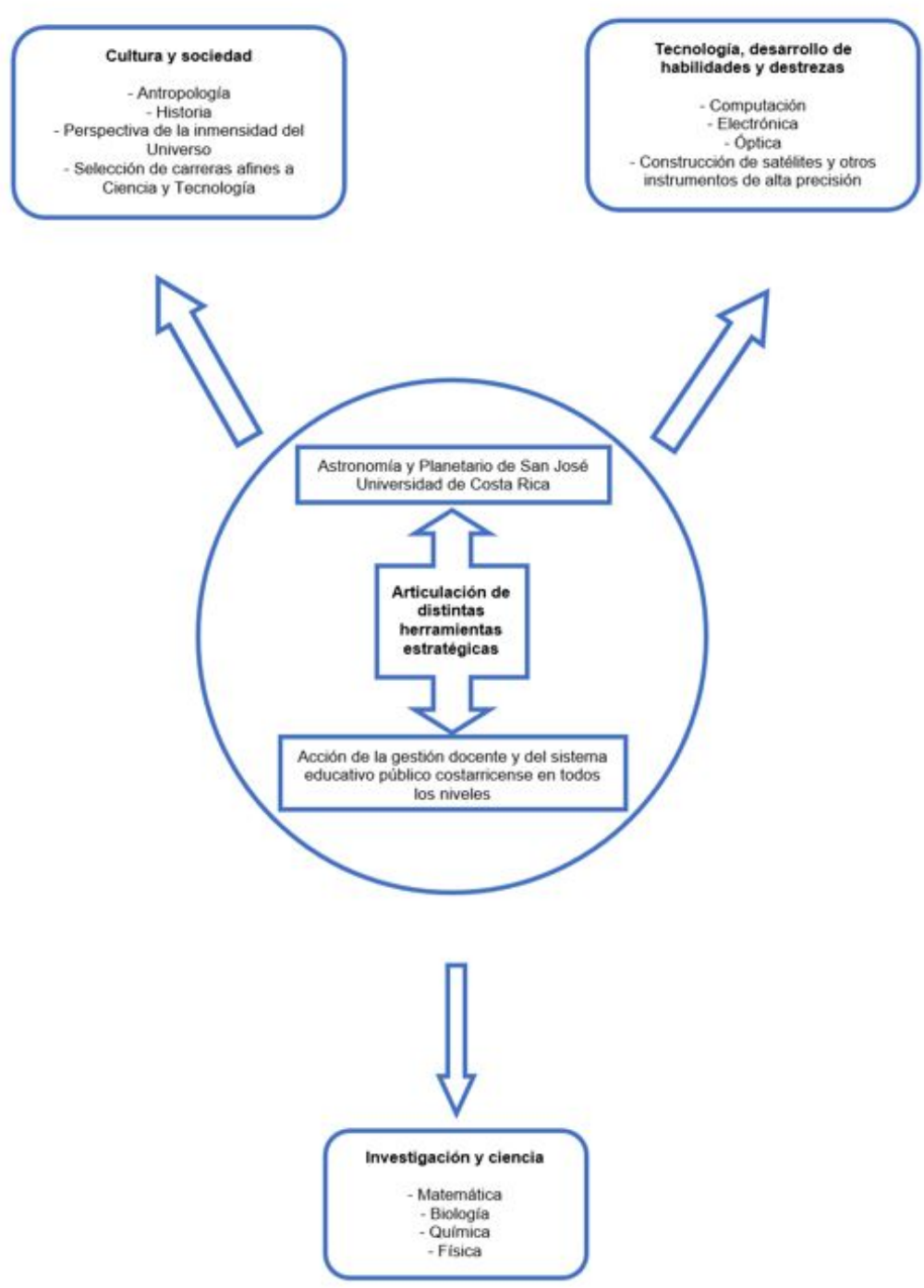

FIGURA 2

Importancia del Planetario de San José de la Universidad de Costa Rica, en el mejoramiento del desarrollo de la Astronomía y el desarrollo sostenible.

Fuente: Elaboración propia, 2016; adaptado del Plan Estratégico 2010-2020 propuesto por la Unión Astronómica Internacional (IAU, 2012).

Es claro que el Planetario de San José es una organización estratégica y factor clave, accesible e indispensable a través de la cual se pueden articular los distintos planteamientos manifestados por la Unión Astronómica Internacional (IAU, 2012), plasmados en el Plan Estratégico antes mencionado, y las demandas señaladas en el Primer Informe sobre el Estado de la Ciencia, la Tecnología y la Innovación 2014 (Programa Estado de la Nación, 2014); con la finalidad de seguir fomentando el interés por la Astronomía, y en consecuencia, contribuir al desarrollo y producción de conocimiento, que a su vez repercutirá, si se aplica ética y correctamente, en el desarrollo científico, tecnológico, educativo, social, político y económico de la sociedad; mejorando al mismo tiempo la calidad de vida de la población en general. 


\section{CONSIDERACIONES FINALES}

La Astronomía en Costa Rica se ha impulsado desde que De Liendo y Goicoechea propició la enseñanza de la Física experimental; y cuyos pasos siguieron Rudín, Pittier, Coen y McGuie; al promover el interés por la física, la matemática y la astronomía; y que en la actualidad continúa consolidando Taliashvili con la coordinación del curso Fundamentos de Astronomía , lo cual es una clara manifestación de la relevancia de la participación e influencia de profesionales extranjeros en nuestro país.

Todos han desarrollado una notable labor en términos de la Administración de la Educación desde distintos ángulos: educativos, científicos, tecnológicos, educativos, sociales, políticos y económicos, entre otros. Actualmente, la importancia de tales aportes se visualiza en la creación y función del Planetario de San José en el sistema educativo costarricense; y en la sociedad en general, a través de su programación diaria, noches de observación o Telescopiadas, talleres astronómicos, conciertos de Música bajo las estrellas, cursos astronómicos, conferencias sobre distintas temáticas, entre otros.

En este sentido, es evidente e indiscutible la importancia de la contribución del Planetario de San José como organización profesional en el acervo cultural de la sociedad, que aumenta aún más con la elaboración de material didáctico de divulgación científica plasmado en películas educativas, calendarios astronómicos, atención de estudiantes de todas las edades y sectores sociales, y público en general. Aunado a esto, el Planetario de San José también es un baluarte fundamental en el desarrollo de proyectos científicos como el Observatorio Astrofísico Solar $O A S$, el Laboratorio de Rayos Cósmicos $L A R C$ y Radioastronomía con sede en Santa Cruz ROSAC. Lo anterior, sin duda consolida al Planetario de San José como miembro de la prestigiosa Sociedad Internacional de Planetarios ( [IPS] International Planetarium Society).

Sin embargo, paralelo a estos aportes, según se ha visto en este artículo, la falta de articulación de los contenidos astronómicos contemplados en los programas de estudio del Ministerio de Educación Pública, es tan solo uno de tantos retos a los cuales se enfrenta la educación costarricense actual, por cuanto no hay un lineamiento sistemático con respecto a la planificación curricular de distintas temáticas astronómicas, tal y como lo sugiere la Unión Astronómica Internacional en su Plan Estratégico (IAU, 2012).

Ante este escenario, el Planetario de San José constituye no solo un referente en materia científicotecnológica, sino también en materia educativa, por cuanto puede proporcionar apoyo en el ámbito docente y planificación curricular astronómica en general. Es evidente que el Planetario de San José es una organización que constituye una herramienta estratégica de la Universidad de Costa Rica que responde de una forma innovadora al desafiante contexto científico-tecnológico de la sociedad costarricense actual.

No obstante, con la finalidad de promover la Astronomía y en consecuencia la Ciencia y la Tecnología como elementos clave para estimular el desarrollo, es importante que cada sector político, económico, social, educativo y científico-tecnológico concreten un profundo análisis de los distintos requerimientos señalados en el Primer Informe sobre el Estado de la Ciencia, la Tecnología y la Innovación 2014 (Programa Estado de la Nación, 2014), para conducirnos al ideal deseado por la Unión Astronómica internacional en su Plan Estratégico, y consolidar a la sociedad costarricense actual en una constante producción y construcción de conocimiento, que lejos de ser un escenario utópico, tiende más bien a garantizar una mejora en la calidad de vida de la ciudadanía en todos los ámbitos y contextos posibles.

\section{ReFERENCIAS}

Bakulin, P.I., Kononovich, E.V. y Moroz, V.I. (1987). Curso de Astronomia General. Moscú: Editorial MIR.

Drucker, P. (1999). La sociedad poscapitalista. Buenos Aires: Sudamericana.

Fernández, W. (1994). Historia del desarrollo de las Ciencias Atmosféricas en la Universidad de Costa Rica: hasta 1995. Revista Ciencia y Tecnología, 18(1), 31-46. 
Fernández, W. (2008). Informe de fin de gestión. Área docente. Responsable función docente. Costa Rica: Universidad de Costa Rica.

González, L. (1976). Historia de la influencia extranjera en el desenvolvimiento educacional y cientifico de Costa Rica. San José: Editorial Costa Rica, Biblioteca Patria.

International Astronomical Union [IAU] (2012). Astronomy for the Developing World Building from the IYA2009: Strategic Plan 2010-2020. Recuperado de https://www.iau.org/static/education/strategicplan_2010-2020.pdf

Luján, J. (2008). La Nueva Guatemala, el espíritu ilustrado en el Reino de Guatemala e hipótesis sobre algunas de sus consecuencias. Diálogos Revista Electrónica de Historia. Volumen especial 2008, 548-562. Recuperado de https: //revistas.ucr.ac.cr/index.php/dialogos/article/download/31184/30909

Ministerio de Educación Pública [MEP] (2003). Programas de Estudio de Física de la Educación Diversificada. San José, Costa Rica: M.E.P. Recuperado de http://www.mep.go.cr/sites/default/files/descargas/programas-de-estudio/ fisica.pdf

Ministerio de Educación Pública [MEP]. (2012). Programas de Estudio de Ciencias Tercer Ciclo de la Educación General Básica. San José, Costa Rica: M.E.P. Recuperado de https://bit.ly/2Ur4uny

Ministerio de Educación Pública [MEP]. (2014a). Programas de Estudio de Ciencias Primer Ciclo de la Educación General Básica. San José, Costa Rica: M.E.P. Recuperado de https://bit.ly/2Vb3D7D

Ministerio de Educación Pública [MEP]. (2014b). Programas de Estudio de Ciencias Segundo Ciclo de la Educación General Básica. San José, Costa Rica: M.E.P. Recuperado de https://bit.ly/2I6faRY

Páez, J. (1994). Aspectos históricos de la astronomía en Costa Rica. Revista Ciencia y Tecnología, 18(1), 51-73.

Plummer, J., y Small, K. (December, 2013). Informal Science Educators' Pedagogical Choices and Goals for Learners: The Case of Planetarium Professionals. Astronomy Education Review, 12(1), 1-16. doi: http://dx.doi.org/10.3 847/AER2013004

Programa Estado de la Nación (2014). Primer Informe del Estado de la Ciencia, la Tecnología y la Innovación 2014 (carpeta de prensa). Recuperado de http://www.estadonacion.or.cr/ecti/assets/carpetadeprensaweb.pdf

Salas C., Páez, J y Herrera, L., (2013) Historia de la Astronomía en Costa Rica [DVD]. Costa Rica: Planetario de San José de la Universidad de Costa Rica.

Segura, A (2015). Propuesta didáctica para fortalecer la enseñanza de la Astronomia, a nivel de primaria en Costa Rica, según programa de Ciencias del Ministerio de Educación Pública. (Tesis de licenciatura inédita). Universidad Estatal a Distancia, San José, Costa Rica

Sistema de Aplicaciones Estudiantiles [SAE]. (2015). Estadísticaspor Sigla - Grupo. Universidad de Costa Rica [UCR]. San José, Costa Rica.

Solano, F. y Páez, J. (1991). Semblanza de un profesor de ciencias: Don Elliot Coen París. En A. Ruiz, Ciencia y Tecnología en la Construcción del Futuro (pp. 248-262). San José, Costa Rica: Ediciones Guayacán.

Taliashvili, L. (2009). Informe anual del Proyecto Planetario de San José de Universidad de Costa Rica. ED-1616. Proyecto de Acción Social sin publicar. Costa Rica: Universidad de Costa Rica, Costa Rica.

Taliashvili, L. (2010). Programa de educación y divulgación cientifica del Planetario de Universidad de Costa Rica (Programa Planetario). Proyecto de Acción Social sin publicar. Costa Rica: Universidad de Costa Rica, Costa Rica.

Taliashvili, L. (2012). Informe anual del Programa de Educación y Divulgación Científica del Planetario de Universidad de Costa Rica (Programa-Planetario).ED-1616. Proyecto de Acción Social sin publicar. Costa Rica: Universidad de Costa Rica, Costa Rica.

Taliashvili, L. (2015). Propuesta de Creación: Unidad Especial de Investigación Planetario de San José Universidad de Costa Rica. Vicerrectoría de Investigación sin publicar. Costa Rica: Universidad de Costa Rica, Costa Rica.

\section{BY-NC-ND}

